\title{
Reagentless $\mathrm{H}_{2} \mathrm{O}_{2}$ Biosensor with High Electron Transfer Efficiency Carrying Nanocomplex of Horseradish Peroxidase and Au-Nanoparticle
}

\author{
Yusuke OKawa, * Yoshinori SakaI, and Fumiyuki SHIBA
}

\begin{abstract}
Graduate School of Advanced Integration Science, Chiba University (1-33 Yayoicho, Inage-ku, Chiba-shi 263-8522, Japan)

Received November 30, 2007 ; Accepted July 15, 2008

A novel electrochemical biosensor for hydrogen peroxide with high electron transfer efficiency was developed using a nanocomplex composed of horseradish peroxidase and Au-nanoparticle. The nanocomplex was prepared through adsorption of horseradish peroxidase on an Au-nanoparticle. The nanocomplexes were easily coagulated and immobilized on an ITO electrode as a monolayer film by simply casting the nanocomplex dispersion and drying below $10^{\circ} \mathrm{C}$. The electrode worked as a hydrogen peroxide biosensor featuring high electron transfer efficiency without any soluble mediator at a mild electrode potential of $+0.15 \mathrm{~V}$ vs. $\mathrm{Ag} / \mathrm{AgCl}$.
\end{abstract}

Key Words : Biosensor, Nanocomplex, Au-nanoparticle, Horseradish Peroxidase, Electron Transfer

\section{Introduction}

Establishment of electronic interface between a redox enzyme and an electrode is one of key techniques for biosensor construction and development of bioelectronics devices. Many types of "third generation" biosensors, that feature direct charge transfer between the enzyme and the electrode and require no soluble electron mediator, have been reported in view of this. ${ }^{1)}$ Recent development of nanomaterials enables us new strategies using nanoparticles for biosensor construction. Xiao et al. reported an $\mathrm{H}_{2} \mathrm{O}_{2}$ biosensor based on an Au-nanoparticle modified Au electrode. Horseradish peroxidase (HRP) was adsorbed on the immobilized Au-nanoparticle and the current due to direct electrochemistry of the adsorbed HRP was observed. ${ }^{2}$ Jia et al. reported a reagentless biosensor for hydrogen peroxide using an electrode carrying HRP-adsorbed Au-nanoparticles incorporated in a three-dimensional silica gel network. ${ }^{3)}$ Xiao et al. reported extraordinarily high electron transfer efficiency for an Au-nanoparticle system combining a sophisticated but rather complicated chemical modification strategy of the nanoparticle surface with immobilized electron mediators and apoglucose oxidase. ${ }^{4)}$ As demonstrated in these works, Au-nanoparticles are expected to present an effective electrochemical interface to enzymatic reactions.

In the present paper, we report a novel electrochemical biosensor with high electron transfer efficiency without any soluble mediator. The sensor carries a coagulated monolayer film of nanocomplexes composed of HRP molecules and an Au-nanoparticle. The film was immobilized through simply casting and drying from the nanocomplex dispersion. The sensor gave direct electrocatalytic current to $\mathrm{H}_{2} \mathrm{O}_{2}$ with high electron transfer efficiency at a mild potential.

\section{Experimental}

A quasi-monodisperse Au-nanoparticle dispersion (average diameter $16 \mathrm{~nm}, \mathrm{CV}=17 \%$, from JEOL JEM1200EX TEM observation, $5.5 \times 10^{15}$ particles $\mathrm{L}^{-1}$ ) was prepared by citrate reduction of chloroauric acid at the boiling temperature.5) A prescribed amount of horseradish peroxidase (HRP, EC 1.11.1.7, Sigma, Type VI) was added to the dispersion at room temperature. Bovine serum albumin (BSA, Wako) was used for comparison. We examined the nanocomplex formation through UVVis spectroscopy and size exclusion chromatography (SEC; column, Shodex Asahipak GS620 × 2; eluent, pH6.5

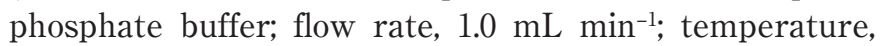
$50^{\circ} \mathrm{C}$; UV detection in the range of $200-520 \mathrm{~nm}$ ). A series of pullulans $\left(\mathrm{MW}=0.59-78.8 \times 10^{4}\right.$, Shodex $)$ were used as the molecular size standards for the SEC experiments. The protein/Au-nanoparticle complex was immobilized onto an indium-tin oxide (ITO) electrode (sheet conductance $10 \Omega$ ) by simply casting the prescribed amount of the complex dispersion (typically $50 \mu \mathrm{L} \mathrm{cm}^{-2}$ ) and drying it below $10^{\circ} \mathrm{C}$. The electrochemical studies were performed in a $\mathrm{pH} 6.5$ phosphate buffer with a conventional three-electrode system at $25^{\circ} \mathrm{C}$.

\section{Results and Discussion}

The addition of the protein (HRP or BSA) drastically improved the colloidal stability of the Au-nanoparticle dispersion. Although the as-prepared dispersion easily coagulates by addition of salts or adsorptive cationic species (e.g. pyrrole), etc, the addition of the protein improved the stability of the dispersion to such treatments. The as-prepared Au-nanoparticles are weakly protected with the adsorbed citrate, and addition of the proteins causes replacement of the citrate protecting layer by the strongly protecting one of the protein,5) and thus resulting in the improved stability.

The plasmon absorption of the as-prepared citrate-pro- 
tected Au-nanoparticle was found at $\lambda_{\max }=520 \mathrm{~nm}$. The addition of HRP resulted in red shift to $\lambda_{\max }=525 \mathrm{~nm}$, suggesting the protein adsorption on the Au-nanoparticle surface. ${ }^{6)}$

The SEC of the mixture of HRP and Au-nanoparticle gave an elution peak at a shorter retention time (i.e. larger solute size, $r . t .=24.0 \mathrm{~min}$ ) than that of free HRP $(r . t .=29.5 \mathrm{~min})$. The peak at r.t. $=24.0 \mathrm{~min}$ features absorptions corresponding to Au-nanoparticle, that is, the plasmon absorption at around $520 \mathrm{~nm}$ and the light scattering in UV region $(\lambda<300 \mathrm{~nm})$. The retention time of $24.0 \mathrm{~min}$ for the present conditions corresponds to the molecular weight of $2.0 \times 10^{5}$ for pullulan, and the corresponding hydrodynamic diameter was estimated as roughly $25-30 \mathrm{~nm}^{7}{ }^{7}$ The eluted substance at r.t. $=24.0$ min should have a similar exclusion size. As the diameter of a globular HRP molecule is estimated as $c a .5 \mathrm{~nm}$, a core of $16 \mathrm{~nm}$ in diameter and a shell of $5 \mathrm{~nm}$ in thickness (i.e. monolayer adsorption) can give a complex of 26 $\mathrm{nm}$ in diameter, being consistent with the chromatographic results. We concluded that an Au-nanoparticle core is surrounded with an adsorbed protein monolayer shell. The protein adsorption on nanoparticle surfaces is well known, however, from a slight different angle of view, this is a spontaneous formation of a nanocomplex composed of protein and a nanoparticle (a sort of selfassembly, in broader sense).

Hereafter, we typically describe the results obtained from the dispersion including $1.5 \times 10^{-5} \mathrm{~mol} \mathrm{~L}^{-1}\left(9.1 \times 10^{16}\right.$ molecules $\mathrm{L}^{-1}$ ) HRP and $5.5 \times 10^{15}$ particles $\mathrm{L}^{-1} \mathrm{Au}$ nanoparticle. We could not detect free HRP from the mixed dispersion, it is seen that the most $(>95 \%)$ of HRP added to the Au-nanoparticle dispersion was adsorbed on the particle surface. Thus, the average number ratio of $\mathrm{HRP} / \mathrm{Au}$ for the nanocomplex was estimated to be 16 from the composition of the mixture.

We applied the above nanocomplex as an electrochemical functional unit for biosensor construction. The nanocomplex dispersion was simply cast on an ITO electrode. The typical aliquot loaded per $1 \mathrm{~cm}^{2}$ contained $2.7 \times 10^{11}$ Au-nanoparticles and $4.5 \times 10^{12} \mathrm{HRP}$ molecules. The electrode was dried below $10^{\circ} \mathrm{C}$, and a gray coagulated film of the nanocomplex was then obtained on the electrode. The film is a monolayer of the nanopaticles from the EM observation, and was sufficiently stable against gentle rinsing with water and the electrochemical operation procedures below.

The electrode carrying the coagulated nanocomplex film was examined with cyclic voltammetry (CV). Figure 1 shows the steady-state voltammograms for the electrode carrying the $\mathrm{HRP} / \mathrm{Au}$-nanoparticle complex film in the presence and absence of $\mathrm{H}_{2} \mathrm{O}_{2}$. The electrode showed surface redox wave at $0.38 \mathrm{~V}$ vs. $\mathrm{Ag} / \mathrm{AgCl}$. Similar peaks were observed for the electrode carrying a similarly prepared electrode carrying a citrate-protected Au-nanoparticle film, and may be assigned for the surface redox of the Au-nanoparticles themselves, although the direct electrochemistry of the HRP redox center may overlap. When $\mathrm{H}_{2} \mathrm{O}_{2}$ was added to the buffer, the catalytic reduction current was observed in the potential

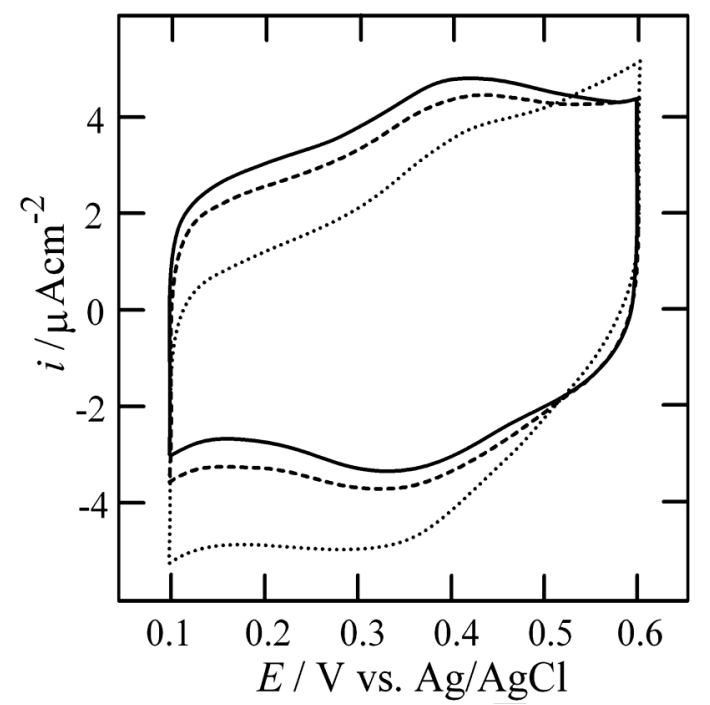

Fig. 1 Steady-state cyclic voltammogram for an electrode carrying an $\mathrm{HRP} / \mathrm{Au}$-nanoparticle complex coagulated film in a pH 6.5 phosphate buffer. $\mathrm{H}_{2} \mathrm{O}_{2}$ concentration; 0 (solid line),

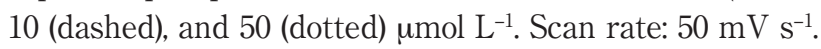

range $<0.45 \mathrm{~V}$ for the $\mathrm{HRP} / \mathrm{Au}$-nanoparticle modified electrode. When BSA/Au-nanoparticle complex was similarly immobilized onto the ITO electrode, the modified electrode exhibited no catalytic reduction current to $\mathrm{H}_{2} \mathrm{O}_{2}$. For the $\mathrm{HRP} / \mathrm{Au}$-nanoparticle systems reported by Xiao et al., ${ }^{2)}$ and Jia $e t a l .{ }^{3)}$ required more negative potentials of $<-0.2 \mathrm{~V}$ vs. $\mathrm{Ag} / \mathrm{AgCl}$ for the electrocatalytic reduction. The present system works at "milder" potential conditions, suggesting more effective electron transfer between the base electrode and the enzyme molecule.

Figure 2 illustrates the response of the electrode carrying the coagulated film of the HRP/Au-nanoparticle complex as a reagentless $\mathrm{H}_{2} \mathrm{O}_{2}$ biosensor at $0.15 \mathrm{~V}$ vs. $\mathrm{Ag} / \mathrm{AgCl}$. The solution was gently stirred with a magnetic stirrer continuously at $c a$. 1 rps. The electrode gave the catalytic cathodic current corresponding to the above $\mathrm{CV}$ experiment to stepwise additions of an $\mathrm{H}_{2} \mathrm{O}_{2}$ standard solution. When the stirring was terminated, the current response gradually decreased, and when the stirring restarted, the current recovered to the original steady-state level. This means that the supply of the substrate $\left(\mathrm{H}_{2} \mathrm{O}_{2}\right)$ to the electrode is one of the rate determining steps of this system, suggesting a rapid charge transfer process between the sensor and the substrate. The working potential is similar to that for an HRP monolayer modified electrode with soluble mediators, ${ }^{9)}$ and is more positive than that of the sensors based on the direct electrochemistry of HRP on Au-nanoparticle reported by Xiao et al. ${ }^{2)}$ and Jia $e t a l .{ }^{3)}$ suggesting more facilitated electronic communication between the Aunanoparticle core and the enzyme redox center. It is also noteworthy that the working potential of the present sensor is so "mild" that nonspecific redox response for practical samples may effectively be minimized.

The sensor that is thermally treated at $110^{\circ} \mathrm{C}$ for $6 \mathrm{~h}$ in air gave negligible response to $\mathrm{H}_{2} \mathrm{O}_{2}$. We also exam- 

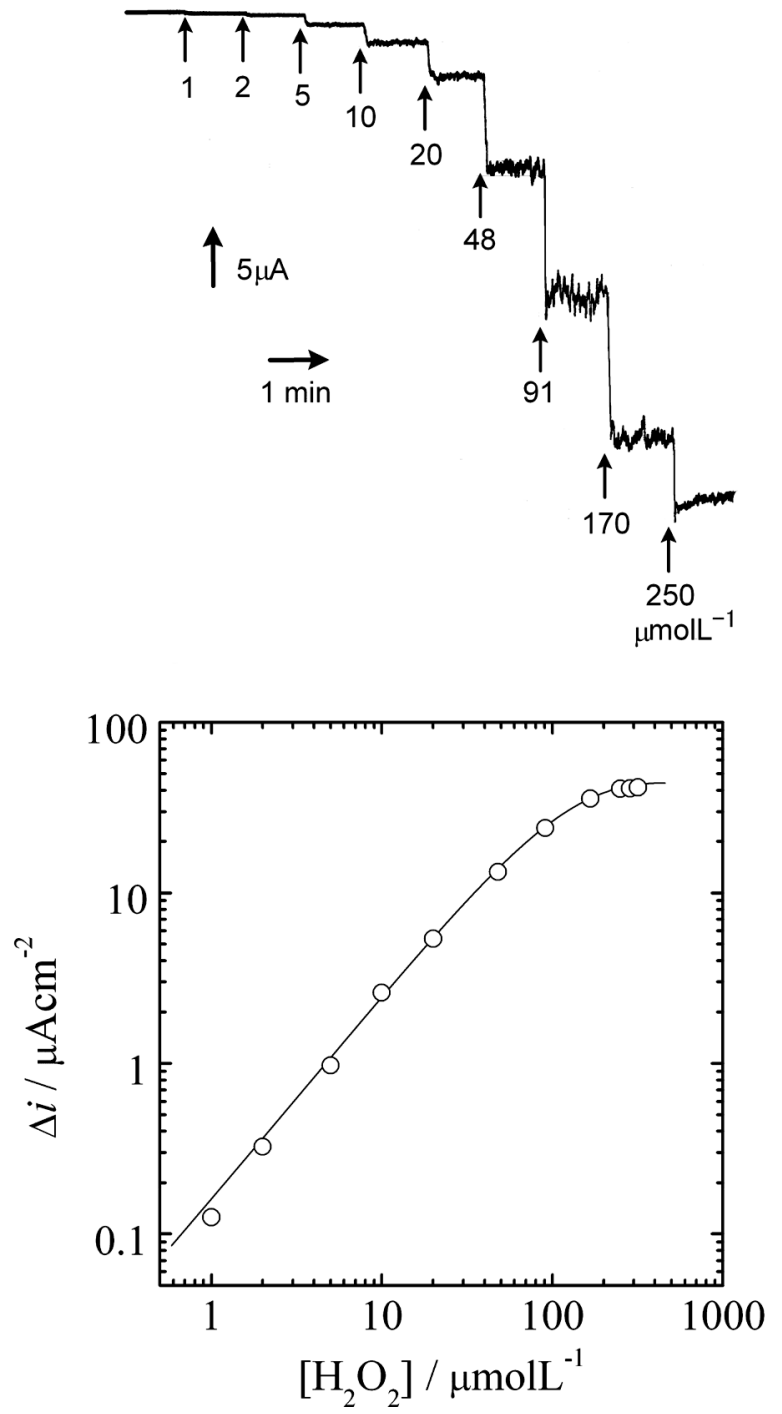

Fig. 2 Typical response of an electrode carrying an $\mathrm{HRP} / \mathrm{Au}$-nanoparticle nanocomplex coagulated film as a reagentless biosensor for $\mathrm{H}_{2} \mathrm{O}_{2}$. A recorder trace for stepwise additions of an $\mathrm{H}_{2} \mathrm{O}_{2}$ standard solution under continuous stirring (upper), and the corresponding calibration graph as a reagentless $\mathrm{H}_{2} \mathrm{O}_{2}$ biosensor (lower). Electrode potential, $0.15 \mathrm{~V}$ vs. $\mathrm{Ag} / \mathrm{AgCl}$.

ined the similarly prepared electrodes carrying the citrate-protected Au-nanoparticles or BSA/Au-nanoparticle complex, and observed negligible response. The response observed in the present study was originated from the enzymatic action of HRP but not from the electrocatalytic activity of the Au-nanoparticles.

Tatsuma et al. reported an HRP monolayer modified electrode system as an $\mathrm{H}_{2} \mathrm{O}_{2}$ biosensor operated with a soluble mediator. ${ }^{9)}$ The sensitivity of the present system for the low substrate concentration region was 40-fold higher than their monolayer-mediator system. They estimated immobilized amounts of HRP as $4.3 \times 10^{12}$ molecules $\mathrm{cm}^{-2}$ at most. This is comparable to the estimated immobilized amount for the present system. Thus, the specific efficiency per enzyme molecule is one order-ofmagnitude higher for the present system, without any soluble mediator. This is partly because of the lack of mediator diffusion process in the present system. Tatsuma et al. also pointed out that the accumulation of the charge-carrying mediator near the electrode surface is of importance in the system of the enzyme monolayer electrode with a soluble mediator through theoretical and numerical simulation analyses. ${ }^{10)}$

The typical enzymatic turnover of the present system was evaluated as $c a .70 \mathrm{~s}^{-1}$ from the saturated sensor output and the amount of HRP loaded. Jia et al. reported electrocatalytic current for an HRP-adsorbed Aunanoparticle system. ${ }^{2}$ They did not state the turnover, however, the estimated one from the data given in the literature is $c a .4 \mathrm{~s}^{-1}$ at $-300 \mathrm{mV}$ vs. $\mathrm{Ag} / \mathrm{AgCl}$. Ruzgas et al. reported the turnover of $0.66 \mathrm{~s}^{-1}$ for the direct electron transfer for an HRP-modified graphite electrode at 0 $\mathrm{mV}$ vs. SCE. ${ }^{11)}$ The value of $70 \mathrm{~s}^{-1}$ for the present system is order-of-magnitude higher than those reported, even under the milder condition $(+150 \mathrm{mV}$ vs. $\mathrm{Ag} / \mathrm{AgCl})$. The present system demonstrates the facilitated electron transport between the enzyme and the electrode through the Au-nanoparticle. Although the mechanism of this high efficiency is unclear to date, it is probably related strongly to the adsorbed state of the enzyme molecules on the Au-nanoparticle and the electronic interaction between the Au-nanoparticle and the enzyme redox center (heme).

We thus concluded that the HRP redox center communicates with the base electrode without any soluble mediator through the Au-nanoparticle core, which works as the nanoscale "connector" between the HRP redox center and the base electrode. The efficiency of the electron transfer in the present system is order-of-magnitude higher than those of the related systems. The HRP/Auparticle nanocomplex was easily immobilized through casting and drying. It is noteworthy that this feature is potentially compatible with sensor preparation using printing technology aiming highly integrated sensor assembly, etc.

\section{References}

1) W. Zhang and G. Li, Anal. Sci., 20, 603 (2004).

2) Y. Xiao, H.X. Ju, and H.-Y. Chen, Anal. Biochem., 278, 22 (2000).

3) J. Jia, B. Wang, A. Wu, G. Cheng, Z. Li, and S. Dong, Anal. Chem., 74, 2217 (2002).

4) Y. Xiao, F. Patolsky, E. Katz, J. F. Hainfeld, and I. Willner, Science (Washington, D. C.), 299, 1877 (2003).

5) J. Turkevich, H. Hillier, and P. C. Stevenson, Discuss. Faraday Soc., 11, 55 (1951); G. Frens, Nature (London) Phys. Sci., 112, 302 (1973).

6) D. Eck, A. Helm, N. J. Wagner, and K. A. Vaynberg, Langmuir, 17, 957 (2001).

7) C. Viebke and P. A. Williams, Anal. Chem., 72, 3896 (2000).

8) T. Vegge, F. Ø. Winther, and B. R. Olsen, Histochemie, 28, 16 (1971).

9) T. Tatsuma, Y. Okawa, and T. Watanabe, Anal. Chem., 61, 2352 (1989).

10) T. Tatsuma and T. Watanabe, Anal. Chem., 64, 625 (1992); T. Tatsuma, T. Watanabe, and Y. Okawa, Anal. Chem., 64, 630 (1992).

11) T. Ruzgas, L. Gorton, J. Emnéus, and G. Marko-Varga, J. Electroanal. Chem., 391, 41 (1995). 